\title{
Journal of Immunobiology
}

\section{Development of de novo Hemolytic Uremic Syndrome Post-Transplant and the Role of Donor Specific Antibodies: A Case Report and Review of Literature}

Sean M Wrenn', Pamela C Gibson², Donna-Sue Hain², Sarah Harm², Paulette B Hammond², Diti H Shah ${ }^{2}$, Susan E Hillyard ${ }^{2}$, Mark Fung ${ }^{2}$ and Carlos E Marroquin ${ }^{*}$

${ }^{1}$ Department of Surgery, Division of Transplant and Hepatobiliary Surgery, University of Vermont College of Medicine, Burlington VT, USA

${ }^{2}$ Department of Pathology, University of Vermont College of Medicine, Burlington VT, USA

\begin{abstract}
Introduction: Hemolytic uremic syndrome (HUS) following kidney transplantation is a devastating complication that may result in substantial morbidity and allograft loss. While calcineurin inhibitor-induced HUS has been well described, anti-donor specific antibody production may be an alternative pathway in the pathogenesis of HUS. We present a case of HUS following kidney transplantation, and will present evidence that donor specific antibodies (DSA) may be a factor in platelet activation and end-organ injury resulting in post-transplant HUS.
\end{abstract}

Case description: A66-year-old female with diabetic nephropathy underwent a deceased donor renal transplant with a $5 \mathrm{HLA}$ mismatched kidney. Her immediate course was uneventful with normalization of her creatinine $(\mathrm{Cr})$. She was re-admitted with rising $\mathrm{Cr}$, oliguria, proteinuria, anemia and thrombocytopenia. A peripheral smear revealed schistocytes, haptoglobin levels were depleted and an allograft biopsy was performed that was suggestive of thrombotic microangiopathy (TMA) with equivocal findings for AMR. Her ADAMTS 13 activity was 103\% (normal). She concurrently developed a substantial de novo DSA burden. She underwent therapeutic plasma-pheresis, conversion from tacrolimus to cyclosporine, and received rituximab therapy. She had a complete clinical resolution and remains off of dialysis. Her laboratory markers improved and her antibody titers decreased.

Discussion: Post-transplant HUS requires immediate recognition and treatment. This clinical course suggests DSA may be involved in an alternative mechanism of platelet activation leading to HUS and renal insult. Review of the literature suggests this is a rare cause of HUS and we postulate may be under-diagnosed in the transplant population and requires further study.

Keywords: Hemolytic uremic syndrome; Donor specific antibodies; Kidney transplant; Allograft rejection

\section{Introduction}

The de novo development of hemolytic uremic syndrome (HUS) following kidney transplantation can be a devastating complication resulting in grave morbidity. Despite a large volume of research regarding HUS, the causes following transplantation are often unclear and multifactorial in nature. While it has previously been established that immunosuppressive agents such as Cyclosporine $\mathrm{A}$ and Tacrolimus can induce HUS, the role of donor specific antibodies (DSA) precipitating post-transplant HUS is a less well established mechanism that may be under-diagnosed in the transplant population [1-3]. We present a case of HUS following kidney transplantation that was treated effectively, and will draw evidence that donor specific antibodies (DSA) may play a role in the pathogenesis of HUS.

\section{Case Description}

A 66 year old female with a past medical history of end stage renal disease presumed secondary to diabetic nephropathy (no kidney biopsy performed), who underwent hemodialysis for 3 years, presented for cadaveric renal transplant. Her major medical co-morbidities included essential hypertension, mild coronary artery disease, prior bleeding gastric ulcer, hypothyroidism, and obesity. Her past surgical history was remarkable for failed arteriovenous fistula, appendectomy, and tonsillectomy. Her prior known sensitizing events included a prior pregnancy, one miscarriage, remote blood transfusion, but no previous transplants. Her relevant family history included diabetes, hypertension, with no known family history of HUS or thrombotic thrombocytopenic purpura (TTP). Her relevant laboratory values pretransplant were serum hemoglobin of 12.9, CMV antibody negative, blood type $\mathrm{B}+$, and calculated panel reactive antibody (PRA) of $55 \%$.
The donor allograft was recovered from a 48 year-old female donor after cardiac death with a terminal creatinine (Cr) of 0.5 , and a 5 HLA mismatch. Total cold and warm ischemia times were 14 hours 9 minutes and 56 minutes, respectively. The left renal allograft was placed in the right retroperitoneal space with a peritoneal window. Given a PRA of 55\%, she received thymoglobulin induction intra-operatively, and was admitted to the surgical intensive care unit in stable condition. She required vasopressor support for the first 36 hours to maintain an adequate mean arterial pressure and was oliguric. On postoperative day two, she started ambulating and was making urine suggestive of graft function. She was transferred out of the intensive care unit on postoperative day three. She developed anemia and thrombocytopenia (platelets to 96) during the immediate post-transplant period likely secondary to Thymoglobulin induction, and ultimately required transfusion of one unit of packed red blood cells on postoperative day five for a hemoglobin of 6.8. She was transitioned to Tacrolimus at the conclusion of the Thymoglobulin induction (total dose $5 \mathrm{mg} / \mathrm{kg}$ ), and was discharged home on postoperative day seven after successfull

*Corresponding author: Carlos E Marroquin, Department of Surgery, Division of Transplant and Hepatobiliary Surgery, University of Vermont College of Medicine, South Prospect Street, Burlington, VT 05401, USA. Tel: (802) 847-3619; Fax: (802) 847-3619; E-mail: Carlos.Marroquin@uvmhealth.org

Received October 27, 2015; Accepted October 30, 2015; Published November 09, 2015

Citation: Wrenn SM, Gibson PC, Hain DS, Harm S, Hammond PB, et al. (2015) Development of de novo Hemolytic Uremic Syndrome Post-Transplant and the Role of Donor Specific Antibodies: A Case Report and Review of Literature. J Immuno Biol 1: 101.

Copyright: (c) 2015 Wrenn SM, et al. This is an open-access article distributed under the terms of the Creative Commons Attribution License, which permits unrestricted use, distribution, and reproduction in any medium, provided the original author and source are credited. 
Citation: Wrenn SM, Gibson PC, Hain DS, Harm S, Hammond PB, et al. (2015) Development of de novo Hemolytic Uremic Syndrome Post-Transplant and the Role of Donor Specific Antibodies: A Case Report and Review of Literature. J Immuno Biol 1: 101.

transition to maintenance immunosuppression with Tacrolimus, Cellcept and Prednisone. She had slow but appropriate graft function (Cr 2.5) with good urine output at discharge and no indication for renal replacement therapy.

Two days after discharge, our recipient suffered a syncopal episode at home as a result of hypoglycemia. She was re-admitted to the transplant service for further evaluation. She underwent an allograft ultrasound, notable only for borderline elevated resistive indices in the vasculature. During her re-admission, her $\mathrm{Cr}$ rose from a nadir of 1.88 to 2.88 . This was initially attributed to either calcineurin inhibitor (Tacrolimus) toxicity, acute rejection, or intravascular volume depletion with pre-renal insult as a result of the circumstances leading to her syncopal episode. Her donor specific antibody levels were rechecked with a single antigen bead solid phase assay (Luminex) and a graft biopsy was performed.

Due to rising creatinine with persistent anemia and thrombocytopenia, there was also a concern for HUS. A serum haptoglobin level, lactate dehydrogenase (LDH) and peripheral blood smear were drawn and evaluated. Her peripheral smear revealed schistocytes, her haptoglobin level was low $(<6 \mathrm{mg} / \mathrm{dl})$, and her LDH was elevated. The ensuing allograft biopsy showed thrombotic microangiopathy (TMA), with mild peri-tubular capillaritis with indeterminate $\mathrm{C} 4 \mathrm{~d}$ immunofluorescence staining $(<50 \%$ positive $)$ consistent with diagnosis of HUS and equivocal for AMR (Figures 1 and 2).

She was treated for HUS with multiple sessions of therapeutic plasma exchange (TPE). Moreover, since there was concern this could represent Tacrolimus-induced HUS, the Tacrolimus was discontinued and she was started on Cyclosporine and continued on Mycophenolate and Prednisone. After five total TPE sessions, her renal function began to improve, LDH continued to trend downward, and her platelets began to normalize. While the anemia eventually resolved, she did require transfusion of two additional units of packed red blood cells (Figure 3).

Her DSA levels were noted to be significantly elevated compared to pre-transplant levels and were positive for anti-A24, anti-B44, anti-B55, and anti-DRB1. Given her substantial change in DSA, we became concerned this could represent an antibody mediated process. As such,

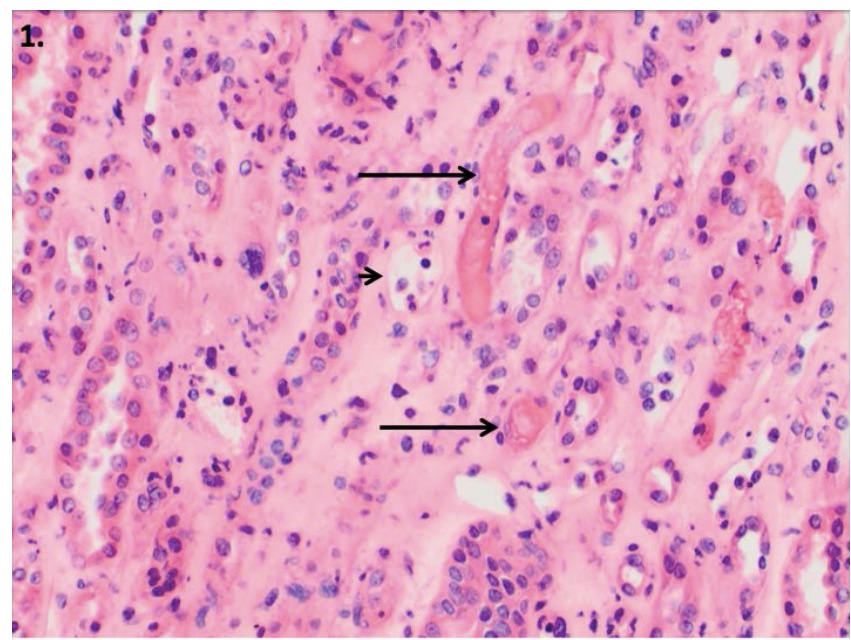

Figure 1: Medullary region of allograft biopsy obtained at onset of HUS symptoms with small vascular thrombi (arrows) and scattered dilated capillaries with peri -tubular capillaritis (short arrow) (200X, H and E Stain).

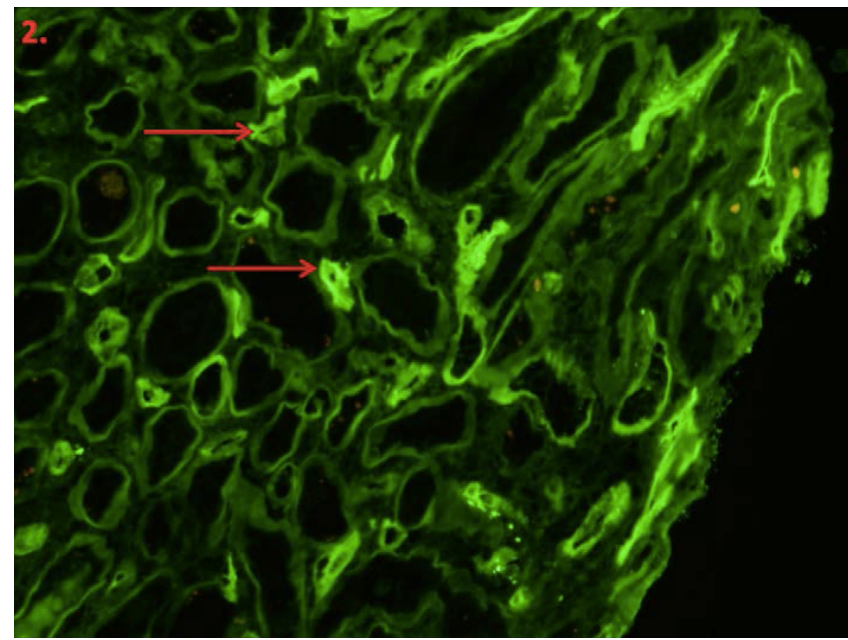

Figure 2: C4d Immunofluorescence staining of less than $50 \%$ of the peritubular capillaries (arrows) (200X, FITC), noted on the initial allograft biopsy.

she was started on intravenous immunoglobulin (IVIG) at completion of TPE for treatment of antibody-mediated rejection (AMR). She also received one dose of Rituximab $\left(375 \mathrm{mg} / \mathrm{m}^{2} \mathrm{IV}\right)$ at the completion of IVIG. Her DSA, serum Cr, Cyclosporine, platelet, and hemoglobin levels were trended closely during her TPE and IVIG. With these interventions, her Cr improved to a new baseline of 1.5. Her DSA levels were drawn on serial dates, and the class I DSA titers (anti-B55 and anti-A24) continued to rise (Figure 4).

On postoperative day 37 , she was noted to have an increasing $\mathrm{LDH}$ and declining platelets, and a peripheral blood smear revealed the presence of schistocytes. She received more TPE and an ADAMTS13 level was checked and found to be $103 \%$ (normal). A repeat renal allograft biopsy was performed, and had features suggestive of TMAwith no histologic features to support the typical AMR with a negative $\mathrm{C} 4 \mathrm{~d}$. There was vacuolization of the tubular epithelial cells with rare foci of moderate tubulitis with mild interstitial inflammation. This resolved after TPE and dosage adjustment of cyclosporine. She was discharged home with stable LDH and Cr levels. At her one year follow-up, she had a stable Cr of 1.38, with low level DSA (solid phase assay: anti-B55/ MFI 3814, anti-A24/MFI 1576, and anti-B44/MFI 597). She has had no additional complications or major hospitalizations since her treatment of her de novo HUS.

\section{Discussion and Review of Literature}

\section{Epidemiology}

Post-transplant thrombotic microangiopathy (TMA) represents a pathology-based diagnosis of the allograft, and encompasses a broad spectrum of disease that includes both HUS and TTP. TMA, as noted on biopsy, is found in $3-14 \%$ of patients receiving calcineurin inhibitor immunosuppression after renal transplant [4]. TMA can either be localized to the allograft or can represent a systemic process. When contained in the renal allograft, it often presents as delayed graft function or acute kidney injury [4]. The frequency of de novo post-kidney transplant HUS presenting as a systemic disorder with severe TMA, is estimated to be $1-5 \%$ [5]. HUS, in contrast to purely localized TMA, presents with hemolytic anemia, thrombocytopenia, in addition to declining renal function [4]. Similar to the findings in our case report, it most often occurs in the first 3 months after transplant $[5,6]$. Transplant TMA, in all forms, has been shown to decrease both 
Citation: Wrenn SM, Gibson PC, Hain DS, Harm S, Hammond PB, et al. (2015) Development of de novo Hemolytic Uremic Syndrome Post-Transplant and the Role of Donor Specific Antibodies: A Case Report and Review of Literature. J Immuno Biol 1: 101.

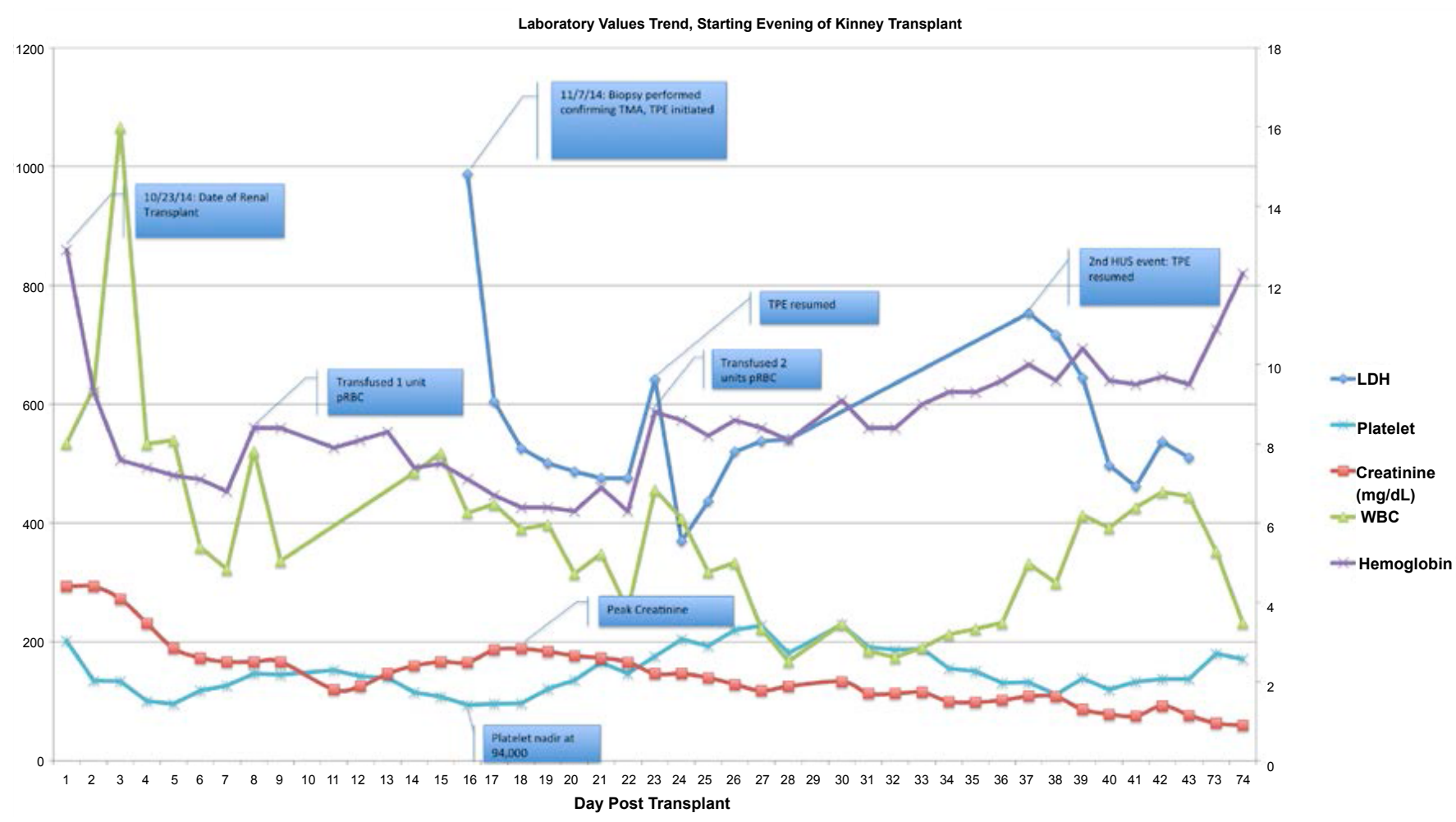

Figure 3: This schematic tracks the most relevant laboratory values over time in her post-transplant course. LDH=Lactate dehydrogenase (U/L), Platelet (1000/cm²), Creatinine $(\mathrm{Cr})(\mathrm{mg} / \mathrm{dl})$, WBC $\left(1000 / \mathrm{cm}^{2}\right)$, Hemoglobin $(\mathrm{gm} / \mathrm{dl})$.

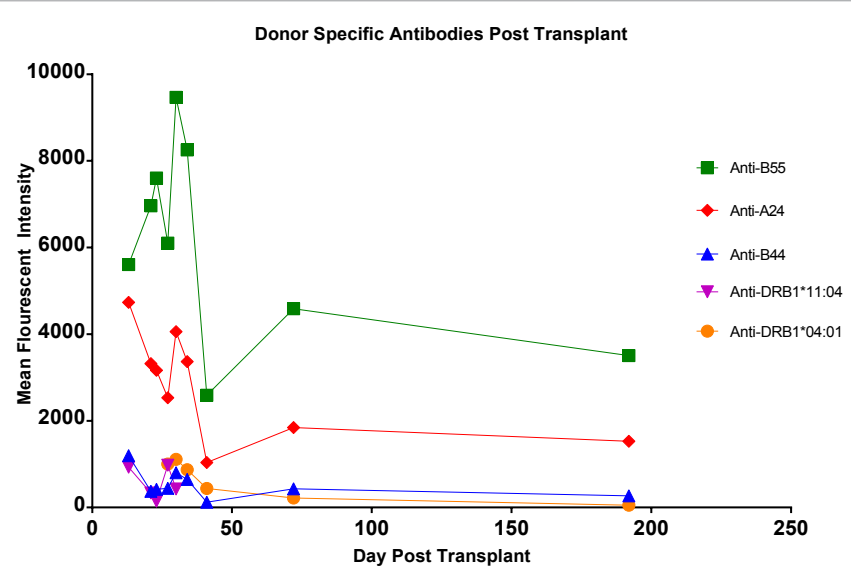

Figure 4: Donor specific antibodies, as measured by solid phase assay (Luminex) in Mean Fluorescent Intensity. The antibodies reach an apparen peak at day 29 post-transplant, with anti-B55 reaching 9,466 MFI. At one year post transplant (not illustrated, MFl's continued to downtrend, though anti-B55, $\mathrm{A} 24$, and B44 remained detectable.

graft and patient survival [7]. The three-year survival rate for posttransplant TMA was reported as $50 \%$, demonstrating the seriousness of the complication [6].

\section{Classification and spectrum of disease}

Hemolytic uremic syndrome (HUS) is not a single disease process, but rather a group of diseases of varied etiologies with a spectrum of manifestations and symptoms. There are multiple useful ways to classify and subdivide etiologies of HUS: atypical vs. typical, "diarrhea-associated" vs. "diarrhea-negative", inherited vs. acquired
[8]. Within the inherited forms of HUS, there can be genetic defects in both complement regulation and inborn errors of metabolism [8]. Additionally, while thrombotic thrombocytopenic purpura (TTP) is now recognized as a distinct disease process, it also has an overlapping presentation with HUS and can have a similar histopathological appearance [8,9]. The hallmark of TTP is a deficiency of serum ADAMTS 13 (less than five percent activity); representing an inability to cleave von Willebrand factor and resulting in pathologic platelet activation and aggregation [9].

\section{Diagnosis}

It is imperative that HUS be identified and treated immediately as it presents a threat to both patient and allograft. As such, it is critical to have an index of suspicion in patients who present with components of HUS; specifically rising $\mathrm{Cr}$ with associated anemia and thrombocytopenia. While serum haptoglobin and LDH levels are easily obtained with a blood smear to corroborate the suspicion, a tissue diagnosis is essential to proper treatment. The histologic evaluation will allow distinction of HUS from classic AMR in the majority of cases.

While both TMA and AMR have long been considered separate clinical entities, AMR has also been thought a potential cause of de novo post-transplant TMA [1]. Both disease processes can be immunemediated and can ultimately manifest with complement deposition in the allograft, resulting in endothelial damage and ultimately graft failure. There appears to be considerable overlap in the pathophysiology of the disease processes between TMA and AMR. The importance of local complement mediated damage has been well established in both processes [8]. Importantly, even with proper and prompt treatment, both increase the risk for short and long term graft failure [1]. 
Citation: Wrenn SM, Gibson PC, Hain DS, Harm S, Hammond PB, et al. (2015) Development of de novo Hemolytic Uremic Syndrome Post-Transplant and the Role of Donor Specific Antibodies: A Case Report and Review of Literature. J Immuno Biol 1: 101.

Diagnostically, it is important to distinguish diarrhea or Shiga associated HUS from atypical HUS (aHUS) and de novo HUS, as they frequently have different etiologies and recurrence patterns. Recurrent renal injury or TMA is rare in patients who developed renal disease due to the diarrheal illness. While aHUS or recurrent TMA is most often a genetic defect in complement regulation and usually the source of renal failure, de novo TMA is seen in patients with no prior history of HUS associated renal injury and most often due to calcineurin inhibitor toxicity, antibody mediated rejection, or viral infection following transplantation [3]. Patients with aHUS usually have one of several genetic mutations resulting in an over-activation of the complement cascade with an unacceptably high recurrence rate. These include Factor $\mathrm{H}, \mathrm{C} 3$, membrane cofactor protein, complement factor B, C322, and Factor I $[3,5]$. If a prior history of HUS is known or suspected, a genetic workup and testing for known mutations is recommended.

Differentiating between AMR and other forms of acute endothelial injury can be difficult because of overlapping features including evidence of thrombotic microangiopathy. Features that help distinguish these entities including the presence of definitive staining for C4d in the allograft peritubular capillaries, the presence of peritubular capillaritis and acute glomerulitis in AMR [10,11]. Some authors also indicate that AMR has a greater extent of the vascular involvement (range of vessels involved is greater in antibody mediated rejection) [2]. In our case, the C4d immunofluorescence staining was in the equivocal range (20-50\%) in the first biopsy and only medulla was available in the biopsy with very focal peritubular capillaritis. The lack of cortex limited the assessment of cortical peritubular capillaritis and acute glomerulitis. The second biopsy showed features suggestive of an active thrombotic microangiopathy, and no histopathologic features of active or chronic antibody mediated rejection including negative $\mathrm{C} 4 \mathrm{~d}$ immunofluorescence.

\section{Pathology and pathophysiology}

TMA is the pathological manifestation of end-organ damage that accompanies HUS. Histological features of TMA include pathological lesions of blood vessel wall thickening, swelling of endothelial cells with detachment from their underlying basement membrane, and intraluminal vessel thrombosis that may cause obstruction of the vessel lumen [12]. Karpman et al. noted that this micro vascular endothelial cell injury is the defining feature of TMA in its relation to systemic HUS [12]. In the classic, Shiga-toxin associated HUS, it is believed that Shiga toxin is internalized by endothelial cells, which allows it to activate itself within the cells. By multiple mechanisms, including cytokine release and endothelial cell damage, the Shiga toxin is able to initiate the cascade of endothelial disruption, platelet clumping, and thrombosis that is the hallmark of TMA [12].

Cyclosporine and Tacrolimus are thought to contribute to TMA via vasoconstriction, endothelial toxicity and pro-thrombotic and anti-fibrinolytic effects $[1,5]$. Both Cyclosporine and Tacrolimus upregulate production of vasoconstrictive factors such as endothelin-1 and angiotensin II, create a pro-coagulant state with possible enhanced platelet aggregation, and have been associated with plasma markers of endothelial damage such as thrombomodulin and von Willebrand factor [5]. While calcineurin inhibitors are classically implicated, other classes of immunosuppressive agents, such as mTOR inhibitors, have also been implicated independently and in conjunction with calcineurin inhibitors [5]. TMA secondary to calcineurin inhibitor toxicity is typically treated by switching to an alternative immunosuppressive agent in conjunction with plasmapheresis [2]. In contrast, if TMA is thought to be secondary to AMR, the standard treatment is plasmapheresis, intravenous immunoglobulin (IVIG), steroid pulse, and Rituximab [2]. Because of the different treatment strategies, it is important to diagnostically distinguish which underlying process is contributing to HUS in the post-transplant patient [1].

Systemic versus localized manifestations of TMA also create an important distinction as each group has distinct characteristics and clinical courses. In a large retrospective review by Schwimmer et al., two distinct groups of TMA after renal transplantation were noted: those with systemic manifestations (62\%) and those with TMA localized to the allograft without systemic symptoms (38\%) [4]. Importantly, the subset and majority of patients who had systemic disease were more likely to have allograft loss and subsequently require dialysis [4]. However systemic manifestations in the post-transplant setting may be difficult to distinguish from other causes. Anemia and thrombocytopenia posttransplant are both often multifactorial in nature, and can frequently be attributed to immune suppression induction agents such as thymoglobulin or anemia of chronic disease [13].

\section{Antibody-mediated rejection and hemolytic uremic syndrome}

While the case presented suggests strongly that the DSA's which developed following the transplant were responsible for the pathology and decline in graft function, as their presence and intensity correlate well with the other clinical findings, the diagnosis of "antibody mediated rejection" was difficult to make due to the C4d-negative nature of the biopsies. The diagnosis of AMR has long been difficult due to lack of specific histo-pathological findings on allograft biopsy that are sufficiently sensitive and specific for AMR [14]. While C4d positive staining is a classic Banff criterion for AMR, there is literature support for AMR that is C4d negative, as in our patient [14]. In 2013 the Banff Criteria was updated to reflect the increasing recognition of C4d negative forms of AMR [15]. The new criteria also list "Acute thrombotic microangiopathy, in the absence of any other cause" as satisfactory histological evidence of AMR [15]. As our case did have histological criteria (TMA), $>10 \%$ C4d in the peri-tubular capillaries, and positive donor specific antibodies, it would satisfy the necessary criteria for AMR according to the 2013 Banff guidelines [15].

A retrospective five-year review from Satoskar et al. was the first to address antibody-mediated rejection (AMR) as a cause of TMA [1]. Interestingly, they found that de novo TMA was associated with AMR in the majority of cases, with $55 \%$ of their biopsies being C4d positive as well [1]. One study reviewing pathology specimens of kidneys with TMA found that $88 \%$ of biopsies had C4d deposition, and $90 \%$ had evidence of activation of the classic complement pathway [16]. This provides further support that complement activation is likely a crucial aspect of the TMA process, just as in AMR. When TMA is present histologically, C4d staining of the peri-tubular capillaries along with serum anti-donor antibodies are the two main diagnostic findings that link TMA and AMR [2].

\section{Genetic factors and predisposition}

While in this particular case a genetic workup was not fully explored, the presence of clear instigating factors and no prior history of HUS strongly suggest this variant of HUS was an acquired or "post-transplant" variant. However one case report by Broeders et al. describes a previously clinically silent polymorphism of Factor $\mathrm{H}$ that manifested as de novo 
Citation: Wrenn SM, Gibson PC, Hain DS, Harm S, Hammond PB, et al. (2015) Development of de novo Hemolytic Uremic Syndrome Post-Transplant and the Role of Donor Specific Antibodies: A Case Report and Review of Literature. J Immuno Biol 1: 101.

post-transplant HUS associated with AMR [17]. Similar to our case, these symptoms occurred approximately 2 weeks after transplant, and presented with systemic HUS symptoms and TMA on allograft biopsy. This cannot be definitely ruled out in our patient, as no investigation into complement activity was performed, however it is important to consider a workup for atypical HUS even when there was no prior history of HUS before transplant [17]. It has been previously demonstrated that a genetic predisposition to atypical HUS can be clinically silent until a "second hit", such as transplant rejection, pregnancy, infections, vaccination occurs [8]. Any of these events are capable of initiating a process of antibodymediated complement deposition, which if unregulated can further progress to classic TMA and HUS.

\section{Treatment}

The correct differentiation between AMR, atypical HUS, or calcineurin inhibitor associated HUS is important in directing management and preventing disease progression with early allograft loss. The treatment of calcineurin inhibitor associated HUS involves decreasing or changing immunosuppression, which can have a detrimental effect in situations of immune mediated rejection. For many years therapeutic plasma exchange was one of the few therapeutic options in HUS, and remains a mainstay of treatment $[8,18]$. It is also an important treatment of antibody-mediated rejection, and is capable of removing both donor specific antibodies and complement components from serum [19]. As plasma exchange is not capable of suppressing DSA production, it is typically accompanied by immunosuppressive agents capable of inhibiting antibody production (such as Rituximab) or therapies to neutralize antibodies (such as IVIG) [19]. In our case, the patient received IVIG, Rituximab, and prompt plasma exchange. The patient was also switched from tacrolimus to cyclosporine due to concerns for tacrolimus-induced HUS.

Another emerging therapy is Eculizumab, a human antibody directed against $\mathrm{C} 5$ capable of inhibiting the complement cascade pathway prior to formation of the membrane attack complex. It has proven to be of clinical use in atypical HUS and has been investigated as a potential therapeutic agent in AMR as well [18]. As more treatment modalities are available for both HUS and AMR, with considerable overlap between therapies, allograft function can be saved with prompt diagnosis and treatment.

\section{Conclusion}

Both antibody-mediated rejection and hemolytic uremic syndrome can occur in the immediate post-transplant period and cause irreparable harm to the allograft and patient. While these are classically two distinct disease processes, there is considerable overlap in the pathophysiology with the common event involving some degree of endothelial and vascular insult. Whether this occurs due to DSA binding a donor antigen, complement activation, vasoactive platelet activation, or direct endothelial injury that results in platelet aggregation producing organ dysfunction is not entirely clear. In our patient, the rapid development of donor-specific HLA antibodies seems to have precipitated allograft TMA with systemic HUS, and allograft injury that resolved as we cleared the DSA. While it is still unclear why some patients who develop DSA are asymptomatic, and others develop classic AMR or HUS, it is important to identify the source of the allograft insult to direct treatment. While it is not surprising when patients with a genetic predisposition present with systemic HUS, it can be a real challenge to identify the trigger in those who present with de novo post-transplant HUS. Our case suggests DSA may be involved in an alternative mechanism of platelet activation leading to HUS and renal insult as a result of AMR. Therefore we propose that TMA and HUS are likely tied to a spectrum with AMR, and that patients with certain risk factors, both genetic and acquired, may be predisposed to this dangerous and systemic variant of allograft rejection.

The previously convenient dichotomies used in classification of HUS and AMR may prove misleading and oversimplifying, as there has proven to be considerable overlap within this spectrum of disease. This case report well illustrates the complexities of the diagnosis, and the importance of a thorough work-up. Fortunately, as in this case, rapid identification of HUS and of the development of DSA lead to prompt and appropriate treatment, and long-term allograft function may still be achieved.

\section{Acknowledgement}

The authors would like to thank the patient from whom this case story originated, our families (especially Lia Nelson MD), the staff of the University Of Vermont Medical Center, the Vermont Antibody Working Group (VAWG), Jonathon Boyson PhD, and Sheila Russell.

\section{References}

1. Satoskar AA, Pelletier R, Adams P, Nadasdy GM, Brodsky S, et al. (2010) De novo thrombotic microangiopathy in renal allograft biopsies-role of antibodymediated rejection. American Journal of Transplantation 10: 1804-1811.

2. Noris M, Remuzzi G (2010) Thrombotic microangiopathy after kidney transplantation. 10: 1517-1523.

3. Nadasdy T (2014) Thrombotic microangiopathy in renal allografts: the diagnostic challenge. Current Opinion in Organ Transplantation 19: 283-292.

4. Schwimmer J, Nadasdy TA, Spitalnik PF, Kaplan KL, Zand MS (2003) De novo thrombotic microangiopathy in renal transplant recipients: a comparison of hemolytic uremic syndrome with localized renal thrombotic microangiopathy Am J Kidney Dis 41: 471-479.

5. Zuber J, Le Quintrec M, Sberro-Soussan R, Loirat C Frémeaux-Bacchi V et al. (2011) New insights into postrenal transplant hemolytic uremic syndrome. Nat Rev Nephrol 7: 23-35.

6. Reynolds JC, Agodoa LY, Yuan CM, Abbott KC (2003) Thrombotic microangiopathy after renal transplantation in the United States. Am J Kidney Dis 42: 1058-1068.

7. Wyatt CM, Dikman S, Sehgal V, Murphy BT, Bromberg JS, et al. (2005) Chronic organizing microangiopathy in a renal transplant recipient. Nephrol Dial Transplant 20: 1734-1737.

8. Kaplan BS, Ruebner RL, Spinale JM, Copelovitch L (2014) Current treatment of atypical hemolytic uremic syndrome. Intractable Rare Dis Res 3: 34-45.

9. Furlan M, Lämmle B (2001) Aetiology and pathogenesis of thrombotic thrombocytopenic purpura and haemolytic uraemic syndrome: the role of von Willebrand factor-cleaving protease. Best Pract Res Clin Haematol 14: 437 454.

10. Colvin RB (2007) Antibody-mediated renal allograft rejection: diagnosis and pathogenesis. J Am Soc Nephrol 18: 1046-1056.

11. Racusen LC, Haas M (2006) Antibody-mediated rejection in renal allografts: lessons from pathology. Clin J Am Soc Nephrol 1: 415-420.

12. Karpman D, Sartz L, Johnson S (2010) Pathophysiology of typical hemolytic uremic syndrome. Semin Thromb Hemost 36: 575-585.

13. Poesen R, Bammens B, Claes K, Kuypers D, Vanrenterghem Y, et al. (2011) Prevalence and determinants of anemia in the immediate postkidney transplant period. Transpl Int 24: 1208-1215.

14. Haas M (2012) Pathologic features of antibody-mediated rejection in renal allografts: an expanding spectrum. Current Opinion in Nephrology and Hypertension 21: 264-271.

15. Haas M (2014) An updated Banff schema for diagnosis of antibody-mediated rejection in renal allografts. Current Opinion in Organ Transplantation 19: 315322.

16. Chua JS, Baelde HJ, Zandbergen M, Wilhelmus S, Van Es LA, et al. 
Citation: Wrenn SM, Gibson PC, Hain DS, Harm S, Hammond PB, et al. (2015) Development of de novo Hemolytic Uremic Syndrome Post-Transplant and the Role of Donor Specific Antibodies: A Case Report and Review of Literature. J Immuno Biol 1: 101.

Page 6 of 6

(2015) Complement Factor C4d Is a Common Denominator in Thrombotic Microangiopathy. J Am Soc Nephrol 26: 2239-2247.

17. Broeders EN, Stordeur P, Rorive S, Dahan K (2014)A 'silent', new polymorphism of factor $\mathrm{H}$ and apparent de novo atypical haemolytic uraemic syndrome after kidney transplantation. BMJ Case Reports.
18. Legendre C, Sberro-Soussan R, Zuber J, Rabant M, Loupy A, et al. (2013) Eculizumab in renal transplantation. 27: 90-92.

19. Sadaka B, Alloway RR, Woodle ES (2013) Management of antibody-mediated rejection in transplantation. Surg Clin North Am 93: 1451-1466. 